\title{
A COHOMOLOGICAL CRITERION FOR SPLITTING OF VECTOR BUNDLES ON MULTIPROJECTIVE SPACE
}

\author{
CHIKASHI MIYAZAKI
}

(Communicated by Lev Borisov)

\begin{abstract}
This paper is devoted to the study of a cohomological criterion for the splitting of a vector bundle on multiprojective space. The criterion extends a result of Ballico-Malaspina towards a generalization of the Horrocks criterion on multiprojective space.
\end{abstract}

\section{INTRODUCTION}

This paper concerns a cohomological criterion for the splitting of vector bundles on multiprojective space. The Horrocks theorem [3] says that a vector bundle $E$ on $\mathbb{P}_{k}^{n}$ is a direct sum of line bundles if and only if $\mathrm{H}^{i}(E(t))=0$ for all $1 \leq i \leq n-1$ and $t$. We will study vector bundles on $X=\mathbb{P}_{k}^{n_{1}} \times \mathbb{P}_{k}^{n_{2}}$. In their paper [1, (1.3), (1.4)] Ballico and Malaspina give a cohomological criterion for vector bundles on multiprojective space; see also [2. We will extend their result towards a framework of a generalization of Horrocks criterion.

Throughout this paper $k$ is an algebraically closed field. Our theorem works on the multiprojective space $X=\mathbb{P}_{k}^{n_{1}} \times \mathbb{P}_{k}^{n_{2}}$. For a coherent sheaf $F$ on $X$, we write $F(a, b)=F \otimes p_{1}^{*} \mathcal{O}_{\mathbb{P}_{k}^{n_{1}}}(a) \otimes p_{2}^{*} \mathcal{O}_{\mathbb{P}_{k}^{n_{2}}}(b)$, where $p_{1}$ and $p_{2}$ are the first and second projections from $X=\mathbb{P}_{k}^{n_{1}} \times \mathbb{P}_{k}^{n_{2}}$ to $\mathbb{P}_{k}^{n_{1}}$ and $\mathbb{P}_{k}^{n_{2}}$ respectively. Our main theorem is the following.

Theorem 1.1. Let $E$ be a vector bundle on $X=\mathbb{P}_{k}^{n_{1}} \times \mathbb{P}_{k}^{n_{2}}$, where $n_{1} \geq 2$ and $n_{2} \geq 2$. The vector bundle $E$ is a direct sum of line bundles of $\mathcal{O}_{X}, \mathcal{O}_{X}(0,1)$, $\mathcal{O}_{X}(0,2), \mathcal{O}_{X}(1,0)$ and $\mathcal{O}_{X}(2,0)$ twisted by line bundles of the form $\mathcal{O}_{X}(\ell, \ell)$ if and only if

$$
\mathrm{H}^{i}\left(E\left(j_{1}+t, j_{2}+t\right)\right)=0
$$

for all integers $i, j_{1}, j_{2}$ and $t$ satisfying that $1 \leq i \leq n_{1}+n_{2}-1,-i \leq j_{1}+j_{2} \leq 0$, $-n_{1} \leq j_{1} \leq 0$ and $-n_{2} \leq j_{2} \leq 0$ except for $\left(i, j_{1}, j_{2}\right)=\left(n_{1},-n_{1}, 0\right),\left(n_{1},-n_{1}+\right.$ $1,0),\left(n_{2}, 0,-n_{2}\right),\left(n_{2}, 0,-n_{2}+1\right)$.

The theorem is obtained from an application of the multigraded CastelnuovoMumford regularity. This idea extends to a possible step towards a classification of ACM vector bundles on multiprojective space.

Received by the editors December 20, 2012 and, in revised form, August 16, 2013.

2010 Mathematics Subject Classification. Primary 14F05, 14J60.

Key words and phrases. Cohomological criterion, Castelnuovo-Mumford regularity.

This work was partially supported by Grant-in-Aid for Scientific Research (C) (21540044) Japan Society for the Promotion of Science. 


\section{Proof of the MAIN THEOREM}

We describe the multigraded Castelnuovo-Mumford regularity according to [1] with some remarks; see also 4. This ingenious definition yields the corresponding property, which will play an important role in the proof of the main result.

Definition 2.1. Let $X=\mathbb{P}_{k}^{n_{1}} \times \mathbb{P}_{k}^{n_{2}}$, where $n_{1} \geq 0$ and $n_{2} \geq 0$. A coherent sheaf $F$ on $X$ is said to be 0 -regular if $\mathrm{H}^{i}\left(F\left(j_{1}, j_{2}\right)\right)=0$ for all integers $i, j_{1}$ and $j_{2}$ such that $i \geq 1, j_{1}+j_{2}=-i,-n_{1} \leq j_{1} \leq 0$ and $-n_{2} \leq j_{2} \leq 0$.

Further, a coherent sheaf $F$ on $X$ is said to be $\left(m_{1}, m_{2}\right)$-regular if $F\left(m_{1}, m_{2}\right)$ is 0 -regular.

Remark 2.2. Let $F$ be a coherent sheaf on $X=\mathbb{P}_{k}^{n_{1}} \times \mathbb{P}_{k}^{n_{2}}$. Assume that $F$ is 0-regular. For a generic hyperplane $H_{1}$ of $\mathbb{P}_{k}^{n_{1}},\left.F\right|_{L_{1}}$ is 0-regular on $L_{1}=H_{1} \times \mathbb{P}_{k}^{n_{2}}$.

Remark 2.3. Let $E$ be a vector bundle on $X=\mathbb{P}_{k}^{n_{1}} \times \mathbb{P}_{k}^{n_{2}}$. Assume that $E$ is 0 -regular. Then $E\left(m_{1}, m_{2}\right)$ is 0-regular for $m_{1} \geq 0, m_{2} \geq 0$. Further, $E$ is globally generated.

Now we will give the proof of our main theorem.

Proof of Theorem 1.1. In order to prove the "only if" part we need to consider the vanishing of the cohomologies $\mathrm{H}^{i}\left(\mathcal{O}_{X}(a, b) \otimes \mathcal{O}_{X}\left(j_{1}+t, j_{2}+t\right)\right)=0$ for $(a, b)=$ $(0,0),(1,0),(0,1),(2,0),(0,2)$. In fact, $\mathrm{H}^{n_{1}}\left(\mathcal{O}_{X}\left(j_{1}+t+a, j_{2}+t+b\right)\right) \neq 0$ if and only if $j_{1}+t+a \leq-n_{1}-1$ and $j_{2}+t+b \geq 0$; in other words, $-j_{2}-b \leq t \leq-n_{1}-j_{1}-a-1$. Similarly, $\mathrm{H}^{n_{2}}\left(\mathcal{O}_{X}\left(j_{1}+t+a, j_{2}+t+b\right)\right) \neq 0$ if and only if $-j_{1}-a \leq t \leq-n_{2}-$ $j_{2}-b-1$. Thus we obtain that if $j_{1}-n_{2}+a-b \leq j_{2} \leq j_{1}+n_{1}+a-b$ for $(a, b)=(0,0),(1,0),(0,1),(2,0),(0,2)$, all the required cohomologies vanish.

We will show the "if" part. There is an integer $t$ such that $E(t, t)$ is 0-regular but $E(t-1, t-1)$ is not 0 -regular.

First assume that $\mathrm{H}^{n_{1}+n_{2}}\left(E\left(-n_{1}+t-1,-n_{2}+t-1\right)\right) \neq 0$. Then we have $\mathrm{H}^{0}\left(E^{\vee}(-t,-t)\right) \neq 0$ by Serre duality, which gives a non-zero map $E(t, t) \rightarrow \mathcal{O}_{X}$. The $(t, t)$-regularity of $E$ implies that $E(t, t)$ is globally generated. Then we have a non-zero map $\oplus \mathcal{O}_{X} \rightarrow E(t, t) \rightarrow \mathcal{O}_{X}$, which must be surjective and split. Hence $\mathcal{O}_{X}$ is a direct summand of $E(t, t)$.

Thus we may assume that $\mathrm{H}^{n_{1}+n_{2}}\left(E\left(-n_{1}+t-1,-n_{2}+t-1\right)\right)=0$. Then we have only to focus on the case of non-vanishing of the $n_{1}$-th cohomologies of the vector bundle $E$ with some twist. The rest of the cases, that is, that of the $n_{2}$-th cohomologies, are similarly proved.

Keeping in mind that $E$ is $(t, t)$-regular but not $(t-1, t-1)$-regular, we have only to consider either the case $\mathrm{H}^{n_{1}}\left(E\left(-n_{1}+t, t-1\right)\right) \neq 0$ or $\mathrm{H}^{n_{1}}\left(E\left(-n_{1}+t-1, t-1\right)\right) \neq 0$ from the assumption. Then we divide into 3 cases:

i) $\mathrm{H}^{n_{1}}\left(E\left(-n_{1}+t, t-1\right)\right) \neq 0$,

ii) $\mathrm{H}^{n_{1}}\left(E\left(-n_{1}+t-1, t-2\right)\right) \neq 0$,

iii) $\mathrm{H}^{n_{1}}\left(E\left(-n_{1}+t, t-1\right)\right)=\mathrm{H}^{n_{1}}\left(E\left(-n_{1}+t-1, t-2\right)\right)=0$ but

$$
\mathrm{H}^{n_{1}}\left(E\left(-n_{1}+t-1, t-1\right)\right) \neq 0 .
$$

For the case i), there is a non-zero element $s$ of $\mathrm{H}^{n_{1}}\left(E\left(-n_{1}+t, t-1\right)\right)$. Let $R_{u}$ be the polynomial ring in $n_{u}+1$ variables over $k$ for $u=1,2$. Let us take the Koszul 
complex

$$
\mathbf{K}_{\mathbf{u} \bullet}: 0 \rightarrow F_{u, n_{u}+1} \rightarrow F_{u, n_{u}} \rightarrow \cdots \rightarrow F_{u, r} \rightarrow \cdots \rightarrow F_{u, 1} \rightarrow F_{u, 0} \rightarrow 0,
$$

where $F_{u, r}$ is a direct sum of ${ }_{n_{u}+1} C_{r}$ copies of $R_{u}(-r)$ for $u=1,2$. Let us consider the exact sequence $p_{1}^{*}\left(\widetilde{\mathbf{K}_{\mathbf{1}}}\right) \otimes E(t+1, t-1)$, that is,

$$
\begin{aligned}
0 & \rightarrow E\left(-n_{1}+t, t-1\right) \rightarrow E\left(-n_{1}+t+1, t-1\right)^{\oplus n_{1}+1} \\
& \rightarrow \cdots \rightarrow E(t-r+1, t-1)^{\oplus n_{1}+1} C_{r} \\
& \rightarrow \cdots \rightarrow E(t, t-1)^{\oplus n_{1}+1} \rightarrow E(t+1, t-1) \rightarrow 0 .
\end{aligned}
$$

In order to construct a surjective map

$$
\varphi: \mathrm{H}^{0}(E(t+1, t-1)) \rightarrow \mathrm{H}^{n_{1}}\left(E\left(-n_{1}+t, t-1\right)\right)
$$

we need to show $\mathrm{H}^{i}(E(t-i+1, t-1))=0$ for $i=1, \cdots, n_{1}$. In fact, we see $\mathrm{H}^{i}(E(t-i+1, t-1))=\mathrm{H}^{i}\left(E(-i+1,-1) \otimes \mathcal{O}_{X}(t, t)\right)=0$ because $-i \leq(-i+1)+$ $(-1) \leq 0,-n_{1} \leq-i+1 \leq 0$ and $-n_{2} \leq-1 \leq 0$. Thus there is a non-zero element $g \in \mathrm{H}^{0}(E(t+1, t-1))$ such that $\varphi(g)=s(\neq 0) \in \mathrm{H}^{n_{1}}\left(E\left(-n_{1}+t, t-1\right)\right)$.

Let us consider the exact sequence

$$
p_{2}^{*}\left(\widetilde{\mathbf{K}_{2}}\right) \otimes E^{\vee}(-t-1,-t+1),
$$

that is, $0 \rightarrow E^{\vee}\left(-t-1,-n_{2}-t\right) \rightarrow E^{\vee}\left(-t-1,-n_{2}-t+1\right)^{\oplus n_{2}+1} \rightarrow \cdots \rightarrow$ $E^{\vee}(-t-1,-t-r+1)^{\oplus n_{2}+1} C_{r} \rightarrow \cdots \rightarrow E^{\vee}(-t-1,-t)^{\oplus n_{2}+1} \rightarrow E^{\vee}(-t-1,-t+1) \rightarrow$ 0 . In order to construct a surjective map

$$
\psi: \mathrm{H}^{0}\left(E^{\vee}(-t-1,-t+1)\right) \rightarrow \mathrm{H}^{n_{2}}\left(E^{\vee}\left(-t-1,-n_{2}-t\right)\right)
$$

we need to show $\mathrm{H}^{i}\left(E^{\vee}(-t-1,-t-i+1)\right)=0$ for $i=1, \cdots, n_{2}$, equivalently $\mathrm{H}^{i}\left(E\left(-n_{1}+t, n_{1}+t-i-2\right)\right)=0$ for $i=n_{1}, \cdots, n_{1}+n_{2}-1$ by Serre duality. In fact, we see

$$
\begin{aligned}
\mathrm{H}^{i}(E & \left.\left(-n_{1}+t, n_{1}+t-2-i\right)\right) \\
\quad & =\mathrm{H}^{i}\left(E\left(-n_{1}+1, n_{1}-i-1\right) \otimes \mathcal{O}_{X}(t-1, t-1)\right)=0
\end{aligned}
$$

because $-i \leq\left(-n_{1}+1\right)+\left(n_{1}-i-1\right) \leq 0,-n_{1} \leq-n_{1}+1 \leq 0$ and $-n_{2} \leq n_{1}-i-1 \leq$ 0 . By taking a dual element $s^{*} \in \mathrm{H}^{n_{2}}\left(E^{\vee}\left(-t-1,-n_{2}-t\right)\right)$ corresponding to $s \in \mathrm{H}^{n_{1}}\left(E\left(-n_{1}+1, t-1\right)\right)$, we have a non-zero element $f \in \mathrm{H}^{0}\left(E^{\vee}(-t-1,-t+1)\right)$ such that $\psi(f)=s^{*}(\neq 0) \in \mathrm{H}^{n_{2}}\left(E^{\vee}\left(-t-1,-n_{2}-t\right)\right)$. The elements $g$ and $f$ can be regarded as elements of $\operatorname{Hom}\left(\mathcal{O}_{X}(0,2), E(t+1, t+1)\right)$ and $\operatorname{Hom}\left(E(t+1, t+1), \mathcal{O}_{X}(0,2)\right)$ respectively. Let us consider the commutative diagram:

$$
\begin{array}{cccc}
\mathrm{H}^{0}(E(t+1, t-1)) \otimes & \mathrm{H}^{0}\left(E^{\vee}(-t-1,-t+1)\right) & \rightarrow & \mathrm{H}^{0}\left(\mathcal{O}_{X}\right) \\
\downarrow & & \downarrow \\
\mathrm{H}^{n_{1}}\left(E\left(t-n_{1}, t-1\right)\right) & \stackrel{\mathrm{H}^{n_{2}}\left(E^{\vee}\left(-t-1,-t-n_{2}\right)\right)}{\rightarrow} & \rightarrow & \mathrm{H}^{n_{1}+n_{2}}\left(\mathcal{O}_{X}\left(-n_{1}-1,-n_{2}-1\right)\right),
\end{array}
$$

where the left vertical map is $\varphi \otimes \psi$ and the right vertical isomorphism gives the canonical element. Thus we have that $f \circ g$ is an isomorphism. Hence $\mathcal{O}_{X}(0,2)$ is a direct summand of $E(t+1, t+1)$.

For the case ii), there is a non-zero element $s$ of $\mathrm{H}^{n_{1}}\left(E\left(-n_{1}+t-1, t-2\right)\right)$. Then we take the corresponding element $s^{*}$ of $\mathrm{H}^{n_{2}}\left(E^{\vee}\left(-t,-n_{2}-t+1\right)\right)$ by Serre duality. As in the case i) we will have surjective maps

$$
\varphi: \mathrm{H}^{0}(E(t, t-2)) \rightarrow \mathrm{H}^{n_{1}}\left(E\left(-n_{1}+t-1, t-2\right)\right)
$$


and

$$
\psi: \mathrm{H}^{0}\left(E^{\vee}(-t,-t+2)\right) \rightarrow \mathrm{H}^{n_{2}}\left(E^{\vee}\left(-t,-n_{2}-t+1\right)\right) .
$$

As in the same procedure by Koszul complex, we have only to prove that $\mathrm{H}^{i}(E(t-i, t-2))=0$ for $1 \leq i \leq n_{1}$ and $\mathrm{H}^{i}\left(E\left(-n_{1}+t-1, n_{1}+t-i-3\right)\right)=0$ for $n_{1} \leq i \leq n_{1}+n_{2}-1$. In fact, we see

$$
\mathrm{H}^{i}(E(t-i, t-2))=\mathrm{H}^{i}\left(E(-i+1,-1) \otimes \mathcal{O}_{X}(t-1, t-1)\right)=0
$$

by assumption because $-i \leq(-i+1)+(-1) \leq 0,-n_{1} \leq-i+1 \leq 0$ and $-n_{2} \leq$ $-1 \leq 0$. On the other hand, we see

$\mathrm{H}^{i}\left(E\left(-n_{1}+t-1, n_{1}+t-i-3\right)\right)=\mathrm{H}^{i}\left(E\left(-n_{1}+1, n_{1}-i-1\right) \otimes \mathcal{O}_{X}(t-2, t-2)\right)=0$

by assumption because $-i \leq\left(-n_{1}+1\right)+\left(n_{1}-i-1\right) \leq 0,-n_{1} \leq-n_{1}+1 \leq 0$ and $-n_{2} \leq n_{1}-i-1 \leq 0$. Then the maps $g$ and $f$ regarded as elements of $\mathrm{H}^{0}(E(t, t-2))$ and $\mathrm{H}^{0}\left(E^{\vee}(-t,-t+2)\right)$ satisfying $\varphi(g)=s$ and $\psi(f)=s^{*}$ give a splitting map from $\mathcal{O}_{X}(-t,-t+2)$ to $E$. Hence $\mathcal{O}_{X}(0,2)$ is a direct summand of $E(t, t)$.

For the case iii), there is a non-zero element $s$ of $\mathrm{H}^{n_{1}}\left(E\left(-n_{1}+t-1, t-1\right)\right)$. As in the case i), to construct a surjective map

$$
\varphi: \mathrm{H}^{0}(E(t, t-1)) \rightarrow \mathrm{H}^{n_{1}}\left(E\left(-n_{1}+t-1, t-1\right)\right)
$$

we need to show $\mathrm{H}^{i}(E(t-i, t-1))=0$ for $i=1, \cdots, n_{1}$. In fact, we see that $\mathrm{H}^{i}(E(t-i, t-1))=\mathrm{H}^{i}\left(E(-i+1,0) \otimes \mathcal{O}_{X}(t-1, t-1)\right)=0$ for $i \neq n_{1}$ because $-i \leq(-i+1)+0 \leq 0,-n_{1} \leq-i+1 \leq 0$ and $-n_{2} \leq 0 \leq 0$. Also, we see $\mathrm{H}^{n_{1}}\left(E\left(-n_{1}+t, t-1\right)\right)=0$ by assumption. Thus there is a non-zero element $g \in \mathrm{H}^{0}(E(t, t-1))$ such that $\varphi(g)=s(\neq 0) \in \mathrm{H}^{n_{1}}\left(E\left(-n_{1}+t-1, t-1\right)\right)$. Similarly, to construct a surjective map

$$
\psi: \mathrm{H}^{0}\left(E^{\vee}(-t,-t+1)\right) \rightarrow \mathrm{H}^{n_{2}}\left(E^{\vee}\left(-t,-t-n_{2}\right)\right)
$$

we need to show $\mathrm{H}^{i}\left(E\left(t-n_{1}-1, t+n_{1}-2-i\right)\right)=0$ for $i=n_{1}, \cdots, n_{1}+n_{2}-1$. In fact, we see $\mathrm{H}^{i}\left(E\left(-n_{1}+t-1, n_{1}+t-i-2\right)\right)=\mathrm{H}^{i}\left(E\left(-n_{1}+1, n_{1}-i\right) \otimes \mathcal{O}_{X}(t-2, t-2)\right)=0$ for $i \neq n_{1}$ because $-i \leq\left(-n_{1}+1\right)+\left(n_{1}-i\right) \leq 0,-n_{1} \leq-n_{1}+1 \leq 0$ and $-n_{2} \leq n_{1}-i \leq 0$. Also, we see $\mathrm{H}^{n_{1}}\left(E\left(t-n_{1}-1, t-2\right)\right)=0$ by assumption. By taking a dual element $s^{*} \in \mathrm{H}^{n_{2}}\left(E^{\vee}\left(-t,-t-n_{2}\right)\right)$ corresponding to

$$
s \in \mathrm{H}^{n_{1}}\left(E\left(t-n_{1}-1, t-1\right)\right),
$$

we have a non-zero element $f \in \mathrm{H}^{0}\left(E^{\vee}(-t,-t+1)\right)$ such that $\psi(f)=s^{*}(\neq 0) \in$ $\mathrm{H}^{n_{2}}\left(E^{\vee}\left(-t,-t-n_{2}\right)\right)$. The elements $g$ and $f$ can be regarded as elements of $\operatorname{Hom}\left(\mathcal{O}_{X}(0,1), E(t, t)\right)$ and $\operatorname{Hom}\left(E(t, t), \mathcal{O}_{X}(0,1)\right)$ respectively. As in the case of i), we have that $f \circ g$ is an isomorphism. Hence $\mathcal{O}_{X}(0,1)$ is a direct summand of $E(t, t)$.

Therefore the assertion is proved.

Remark 2.4. Summing up the proof, we should note that an essential point is to use the assumption $\mathrm{H}^{n_{1}}(F(1,0))=\mathrm{H}^{n_{1}}(F(0,-1))=0$ for a vector bundle $F$ with $\mathrm{H}^{n_{1}}(F) \neq 0$. Then $F$ has a direct summand $\mathcal{O}_{X}\left(-n_{1}-1,0\right)$. In fact, we applied this procedure to the cases i) $F=E\left(-n_{1}+t, t-1\right)$, ii) $F=E\left(-n_{1}+t-1, t-2\right)$ and iii) $F=E\left(-n_{1}+t-1, t-1\right)$. 
Finally we state a general result proved similarly as in Theorem 1.1

Theorem 2.5. Let $E$ be a vector bundle on $X=\mathbb{P}_{k}^{n_{1}} \times \mathbb{P}_{k}^{n_{2}}$, where $n_{1} \geq 1$ and $n_{2} \geq 1$. Let $r_{1}$ and $r_{2}$ be integers such that $0 \leq r_{1} \leq n_{1}$ and $0 \leq r_{2} \leq n_{2}$. The vector bundle $E$ is a direct sum of line bundles of $\mathcal{O}_{X}, \mathcal{O}_{X}(0,1), \cdots, \mathcal{O}_{X}\left(0, r_{1}\right)$, $\mathcal{O}_{X}(1,0), \cdots \mathcal{O}_{X}\left(r_{2}, 0\right)$ twisted by line bundles of the form $\mathcal{O}_{X}(\ell, \ell)$ if and only if

$$
\mathrm{H}^{i}\left(E\left(j_{1}+t, j_{2}+t\right)\right)=0
$$

for all integers $i, j_{1}, j_{2}$ and $t$ satisfying that $1 \leq i \leq n_{1}+n_{2}-1,-i \leq j_{1}+j_{2} \leq 0$, $-n_{1} \leq j_{1} \leq 0$ and $-n_{2} \leq j_{2} \leq 0$ except for either $i=n_{1}$ and $j_{2} \geq j_{1}+n_{1}-r_{1}+1$, or $i=n_{2}$ and $j_{2} \leq j_{1}-n_{2}+r_{2}-1$.

Outline of the Proof of Theorem 2.5. The "only if" part is easily shown by calculating cohomologies.

In order to show the "if" part we take the minimal integer $t$ such that $E(t, t)$ is 0 -regular. In the case $\mathrm{H}^{n_{1}+n_{2}}\left(E\left(-n_{1}+t-1,-n_{2}+t-1\right)\right) \neq 0$, we see that $\mathcal{O}_{X}$ is a direct summand of $E(t, t)$ as in the proof of Theorem 1.1. In particular, the case $r_{1}=r_{2}=0$ is done. So we may assume that $\mathrm{H}^{n_{1}+n_{2}}\left(E\left(-n_{1}+t-1,-n_{2}+t-1\right)\right)=$ 0 . From the assumption possible non-vanishing parts for the non-0-regularity of $E(t-1, t-1)$ appear in the $n_{1}$-th or $n_{2}$-th cohomologies. Thus we have only to consider the set of pairs $\left(j_{1}, j_{2}\right)$ with $\mathrm{H}^{i}\left(E\left(j_{1}+t, j_{2}+t\right)\right) \neq 0$ for $i=n_{1}, n_{2}$.

Let us put $\mathfrak{S}=\left\{\left(j_{1}, j_{2}\right) \mid j_{1} \geq-n_{1}-1, j_{1}+n_{1}-r_{1}+1 \leq j_{2} \leq-j_{1}-n_{1}-1\right\}$. Note that $\mathfrak{S}$ is non-empty if $r_{1} \geq 1$, and we have $-n_{1} \leq j_{1} \leq-n_{1} / 2-1$ and $-n_{1} \leq j_{2} \leq-1$ for $\left(j_{1}, j_{2}\right) \in \mathfrak{S}$. Since $E(t-1, t-1)$ is not 0 -regular, we may assume there are some $\left(j_{1}, j_{2}\right) \in \mathfrak{S}$ such that $\mathrm{H}^{n_{1}}\left(E\left(j_{1}+t, j_{2}+t\right)\right) \neq 0$. If not, there are non-vanishing $n_{2}$-th cohomologies and then we can proceed similarly. For pairs $\left(j_{1}, j_{2}\right)$ and $\left(k_{1}, k_{2}\right)$ in $\mathfrak{S}$ we define a total order $\left(j_{1}, j_{2}\right)<\left(k_{1}, k_{2}\right)$ if either i) $j_{2}-j_{1}<k_{2}-k_{1}$ or ii) $j_{2}-j_{1}=k_{2}-k_{1}$ and $j_{1}<k_{1}$. For example, in the case of (1.1), $\mathfrak{S}$ consists of $\left(-n_{1}-1,-2\right),\left(-n_{1}-1,-1\right)$ and $\left(-n_{1},-1\right)$ in the increasing order.

Now, back to the proof, we take the minimal $\left(j_{1}, j_{2}\right) \in \mathfrak{S}$ such that

$$
\mathrm{H}^{n_{1}}\left(E\left(j_{1}+t, j_{2}+t\right)\right) \neq 0 .
$$

By taking $F=E\left(j_{1}+t, j_{2}+t\right)$, we have $\mathrm{H}^{n_{1}}(F) \neq 0$ and $\mathrm{H}^{n_{1}}(F(1,0))=$ $\mathrm{H}^{n_{1}}(F(0,-1))=0$ from the minimality of $\left(j_{1}, j_{2}\right)$ in $\mathfrak{S}$ and the assumption. Then the corresponding proof of Theorem [1.1 works for $F=E\left(j_{1}+t, j_{2}+t\right)$ as described in Remark 2.4. Indeed, by using $\mathrm{H}^{n_{1}}(F(1,0))=0$, we have a surjective map $\varphi: \mathrm{H}^{0}\left(F\left(n_{1}+1,0\right)\right) \rightarrow \mathrm{H}^{n_{1}}(F)$. Similarly, by using $\mathrm{H}^{n_{1}}(F(0,-1))=0$, we have a surjective map $\psi: \mathrm{H}^{0}\left(F^{\vee}\left(-n_{1}-1,0\right)\right) \rightarrow \mathrm{H}^{n_{2}}\left(F^{\vee}\left(-n_{1}-1,-n_{2}-1\right)\right)$. Then there are elements $f \in \mathrm{H}^{0}\left(F\left(n_{1}+1,0\right)\right)$ and $g \in \mathrm{H}^{0}\left(F^{\vee}\left(-n_{1}-1,0\right)\right)$ such that $\varphi(f) \otimes \psi(g)$ gives a canonical element via

$$
\mathrm{H}^{n_{1}}(F) \otimes \mathrm{H}^{n_{2}}\left(F^{\vee}\left(-n_{1}-1,-n_{2}-1\right)\right) \rightarrow \mathrm{H}^{n_{1}+n_{2}}\left(\mathcal{O}_{X}\left(-n_{1}-1,-n_{2}-1\right)\right) .
$$

Thus the corresponding maps $f: \mathcal{O}_{X} \rightarrow F\left(n_{1}+1,0\right)$ and $g: F(n+1,0) \rightarrow \mathcal{O}_{X}$ satisfy that $g \circ f$ is an isomorphism. Hence $\mathcal{O}_{X}\left(-j_{1}-n_{1}-1,-j_{2}\right)$ is a direct summand of $E(t, t)$. Repeating this procedure, therefore, yields the assertion.

\section{ACKNOWLEDGMENT}

The author would like to thank the referee for helpful comments. 


\section{REFERENCES}

[1] Edoardo Ballico and Francesco Malaspina, Regularity and cohomological splitting conditions for vector bundles on multiprojective spaces, J. Algebra 345 (2011), 137-149, DOI 10.1016/j.jalgebra.2011.08.015. MR2842058

[2] L. Costa and R. M. Miró-Roig, Cohomological characterization of vector bundles on multiprojective spaces, J. Algebra 294 (2005), no. 1, 73-96, DOI 10.1016/j.jalgebra.2005.08.035. MR2179715 (2007c:14042)

[3] G. Horrocks, Vector bundles on the punctured spectrum of a local ring, Proc. London Math. Soc. (3) 14 (1964), 689-713. MR0169877 (30 \#120)

[4] Diane Maclagan and Gregory G. Smith, Multigraded Castelnuovo-Mumford regularity, J. Reine Angew. Math. 571 (2004), 179-212, DOI 10.1515/crll.2004.040. MR2070149 (2005g:13027)

[5] David Mumford, Lectures on curves on an algebraic surface, With a section by G. M. Bergman. Annals of Mathematics Studies, No. 59, Princeton University Press, Princeton, N.J., 1966. MR0209285 (35 \#187)

Department of Mathematics, Saga University, Honjo-machi 1, Saga 840-8502, Japan

E-mail address: miyazaki@ms.saga-u.ac.jp 